\title{
The Gray GM(1.1) Prediction in Wheat Production of Shandong Province Based on the MATLAB
}

\author{
Qianqian Zhang ${ }^{1, \mathrm{a}}$, Jie Yang ${ }^{2, \mathrm{~b}}$, Yingchun Zhang, ${ }^{1, \mathrm{c}}$, Mingchuan $\mathrm{Ji}^{2, \mathrm{~d} *}$ \\ ${ }^{1}$ School of Electrical Engineering and Automation,Qilu University of Technology. \\ Jinan, China \\ ${ }^{2}$ Shandong Institute of Agriculture Sustainable Development \\ Jinan, China \\ azhqq_520@163.com, ,amber1026@163.com, ‘zyc_10@126.com,jimc6668@163.com
}

\begin{abstract}
MATLAB is a kind of software which has powerful computing capabilities. This paper used the MATLAB software to computing the gray GM(1.1) prediction model, and establish the gray GM(1.1) prediction model based on historical data of production of wheat in Shandong Province. Through residual test, the prediction model is suitable for wheat prediction. This paper predicted the planting area, yield and output of wheat in Shandong Province from 2013 to 2015, and calculated the error range.
\end{abstract} predict

Keywords- MATLAB; gray GM(1.1); wheat production;

\section{INTRODUCTION}

Shandong province is one of the major wheat producing provinces. Wheat acreage was $14.0 \%$ of the country's total wheat acreage, total production accounted for about $16.8 \%$. Shandong Province is not only one of China's main winter wheat producing areas, but also is one of the main producing areas of China's wheat yield and advantages. Forecasting for wheat production in Shandong Province, including its acreage, production and yield, has great significance to promote the development of modern agriculture industrial as well as establish and improve the modern agricultural industrial system. Therefore, we can use MATLAB's powerful data processing capabilities, through preparation gray GM (1.1) forecasting model of wheat production in Shandong Province, to predict the future development of wheat in Shandong Province. Furthermore, MATLAB algorithm reuse rate is very high and improved the efficiency of computing processing.

\section{GRAY GM(1.1) FORECASTING MODEL}

Gray system is differential equation built on a discrete sequence. GM(1.1) is a model of a first-order differential equations. By the generated mechanism of GM(1.1) albino equation, derived its time response sequence as Eq.1, Eq.2 is the reduction value formula.

$$
\begin{gathered}
\hat{x}^{(1)}(k)=\left(x^{0}(1)-\frac{b}{a}\right) e^{-a(k-1)}+\frac{b}{a} \\
\hat{\mathrm{X}}^{(0)}(k+1)=\left(1-e^{a}\right)\left(x^{0}(1)-\frac{b}{a}\right) e^{-a k}
\end{gathered}
$$

Where, -a is development factor, b is gray action. -a reflects the development momentum of $\mathrm{x} \wedge((1))$ and $\mathrm{x}$

$\wedge((0))$. b is exhumed from the background value data, which reflects the data changes, the exact meaning is ash.

The next is model building processing. The original data is as shown in table1.

Step 1: Make the original sequence $X^{\wedge}((0))$ for an accumulative sequence generated 1-AGO sequence $\mathrm{X}^{\wedge}((1))$.

Step 2: According to Eq.3, the mean sequence of the $\mathrm{X}^{\wedge}((1))$ is generated as the sequence $\mathrm{Z}^{\wedge}((1))$.

$$
z^{(1)}(k)=0.5 x^{(1)}(k)+0.5 x^{(1)}(k+1)
$$

Step 3: According to Eq.4 and Eq.5, do least squares estimation to parameters listed $\hat{a}=\left[\begin{array}{l}a \\ b\end{array}\right]$.

$$
\begin{gathered}
\mathrm{a}=\frac{\frac{1}{n-1} \sum_{k=2}^{n} x^{(0)}(k) \sum_{k=2}^{n} z^{(1)}(k)-\sum_{k=2}^{n} x^{(0)}(k) z^{(1)}(k)}{\sum_{k=2}^{n} \llbracket\left(z \rrbracket^{(1)}(k)\right)^{2}-\frac{1}{(n-1)\left(\sum_{k=2}^{n} \llbracket z^{(1)}(k)\right) \rrbracket^{2}}} \\
\mathrm{~b}=\frac{1}{n-1}\left[\sum_{k=2}^{n} x^{(0)}(k)+a \sum_{k=2}^{n} z^{(1)}(k)\right]
\end{gathered}
$$

Step 4: Determine the model expression (as shown in Eq.6) and the time response type (as shown in Eq.7).

$$
\begin{gathered}
\frac{d x^{(1)}}{d t}-a x^{(1)}=b \\
\hat{\mathrm{X}}^{(0)}(k)=\left(1-e^{a}\right)\left(x^{0}(1)-\frac{b}{a}\right) e^{-a(k-1)}
\end{gathered}
$$

Step 5: According to Eq.2, restore and calculate $\mathrm{X}^{\wedge}((0))$ analog values.

Step 6: Choose the relative error test method to test error, calculate the residual sum of squares and mean relative error.

Step 7: Calculate the three-step forecast data.

\section{MATLAB PRogram OF GM(1.1) ForeCASTING MODEL}

The main MATLAB program is as shown in table 2. 


\section{CONCLUSION}

This paper has established gray GM(1.1) models of wheat acreage, production, yields of Shandong Province and the national based on the data from 1999 to 2011. The modeling analysis results are shown in table 3 , the predicted results shown in table 4.

After the modeling analysis of Shandong Province and the country's wheat production data of 1999-2011 years, the statistics of wheat has been completed in 2012 . Therefore, the 2012 forecast data was compared with the actual data to calculate the error, which was as shown in table 4.

Combining the average relative error in table 3 and the analysis results in table5, we can see that the relative error of prediction data obtained by GM (1.1) model is smaller than actual data, within the modeling analysis expected range. Therefore, we can use GM (1.1) model to predict the future of wheat yield. As the forecast data only for the production data of wheat, and wheat production process will be affected by climate, weather, natural disasters, etc.
So in the actual analysis process can also combine the year's environment and climate prediction of wheat growing to get a more accurate predictive analysis.

\section{ACKNOWLEDGMENT}

About the paper: This research comes from the project: Shandong province modern agricultural industry technology system of wheat.

*Mingchuan Ji: Male, Shandong. Juncheng. The secondary researcher. Long engaged in agricultural economics research.

\section{REFERENCES}

[1] Sifeng Liu, Yaoguo Dang, Zhigeng Fang, Naiming Xie. Gray system theory and its application[M].5.Beijing:Science Press, 2010.5.

[2] Buxiang Wang.Prediction of grain yield in China[D].Jiangsu University, 2009.

[3] HenselMan(America),Littlefield(America).RenfengZhu(translated) .Proficient in Matlab 7[M].1.Tsinghua university press, 2006.5.K. Elissa, "Title of paper if known,” unpublished.

TABLe I 1999 2011 Shandong Province AND the Whole Nation Wheat Production Statistic Data

\begin{tabular}{|c|c|c|c|c|c|c|}
\hline \multirow{2}{*}{ year } & \multicolumn{3}{|c|}{ The whole nation } & \multicolumn{3}{c|}{ Shandong Province } \\
\cline { 2 - 7 } & Acreage & production & Yield & Acreage & production & Yield \\
\cline { 2 - 7 } & $\begin{array}{c}\text { one thousand } \\
\text { hectares }\end{array}$ & million tons & kg/hectare & $\begin{array}{c}\text { one thousand } \\
\text { hectares }\end{array}$ & million tons & $\mathrm{kg} /$ hectare \\
\hline 1999 & 28855 & 11388 & 3947 & 4007 & 2117.7 & 5285 \\
\hline 2000 & 26653 & 9963.6 & 3738 & 3960 & 1860 & 4697 \\
\hline 2001 & 24664 & 9387.3 & 3806 & 3546 & 1655.2 & 4668 \\
\hline 2002 & 23908 & 9029 & 3777 & 3397 & 1547.1 & 4554 \\
\hline 2003 & 21997 & 8648.8 & 3932 & 3105 & 1565 & 5040 \\
\hline 2004 & 21626 & 9195.2 & 4252 & 3106 & 1584.6 & 5102 \\
\hline 2005 & 22793 & 9744.5 & 4275 & 3279 & 1800.5 & 5492 \\
\hline 2006 & 23613 & 10846.6 & 4593 & 3557 & 2013 & 5660 \\
\hline 2007 & 23721 & 10929.8 & 4608 & 3519 & 1995.6 & 5671 \\
\hline 2008 & 23617 & 11246.4 & 4762 & 3525 & 2034.2 & 5770 \\
\hline 2009 & 24291 & 11511.5 & 4739 & 3545 & 2047.3 & 5775 \\
\hline 2010 & 24257 & 11518.1 & 4748 & 3561.87 & 2058.6 & 5780 \\
\hline 2011 & 24270 & 11740.1 & 4837 & 3593.5 & 2103.9 & 5855 \\
\hline
\end{tabular}

TABLE II THE MAIN MATLAB PROGRAM

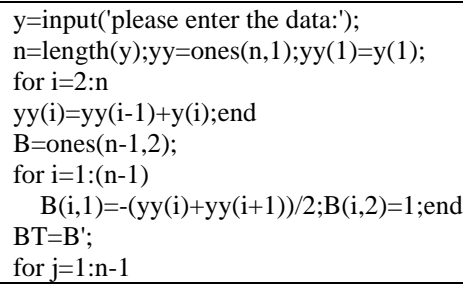




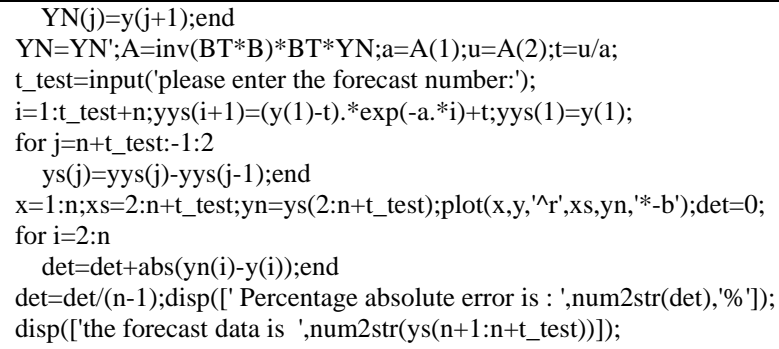

TABle III Shandong And Nation Wheat GM(1.1) Prediction Model Parameter TABle in 1999-2011

\begin{tabular}{|c|c|c|c|c|c|}
\hline \multirow{2}{*}{$\begin{array}{c}\text { Shandong } \\
\text { Province }\end{array}$} & $\begin{array}{c}\text { Wheat } \\
\text { Production }\end{array}$ & $\begin{array}{c}\text { Development } \\
\text { coefficient(-a) }\end{array}$ & $\begin{array}{c}\text { Gray action } \\
\text { (b) }\end{array}$ & $\begin{array}{c}\text { Residual } \\
(\varepsilon)\end{array}$ & $\begin{array}{c}\text { The average } \\
\text { relative error }\end{array}$ \\
\cline { 2 - 6 } & $\begin{array}{c}\text { Yield } \\
\text { (kg/hectare) }\end{array}$ & 0.0237 & 4490.2082 & 36663.0249 & $2.6356 \%$ \\
\cline { 2 - 6 } & Acreage(one thousand hectares) & 0.0008 & 3454.7085 & 594411.8515 & $4.7505 \%$ \\
\hline \multirow{2}{*}{$\begin{array}{c}\text { Whole } \\
\text { nation }\end{array}$} & $\begin{array}{c}\text { Production } \\
\text { (million tons) }\end{array}$ & 0.0261 & 1524.6503 & 182806.2172 & $5.4031 \%$ \\
\cline { 2 - 6 } & $\begin{array}{c}\text { Yield } \\
\text { (kg/hectare) }\end{array}$ & 0.0261 & 3591.8277 & 169190.0096 & $2.2379 \%$ \\
\cline { 2 - 6 } & Acreage(one thousand hectares) & -0.00181 & 24098.2842 & 18370964.789 & $3.7499 \%$ \\
\hline \multirow{2}{*}{$\begin{array}{c}\text { Production } \\
\text { (million tons) }\end{array}$} & 0.0260 & 8487.2551 & 3304413.0259 & $4.3357 \%$ \\
\hline
\end{tabular}

Table IV Shandong and National Wheat Gray GM(1.1) Prediction Model and Predictive Value of the Next Three Years

\begin{tabular}{|c|c|c|c|c|c|c|}
\hline & \multirow{2}{*}{ wheat } & \multirow{2}{*}{ GM(1,1) model time responsive } & \multirow{2}{*}{ Reducing value calculation formula } & \multicolumn{3}{|c|}{ predictive value of the next three years } \\
\hline & & & & 2012 & 2013 & 2014 \\
\hline \multirow{3}{*}{0} & yield(kg/hectare) & $\begin{array}{l}\hat{x}^{(1)}(k) \\
=194745.2616 e^{0.0237(k-1)} \\
-189460.2619\end{array}$ & $\begin{array}{l}\hat{x}^{(0)}(k+1) \\
=4561.2323 e^{0.0237 k}\end{array}$ & 6208.0036 & 6356.9622 & 6509.4951 \\
\hline & $\begin{array}{c}\text { Acreage } \\
\text { (one thousand hectares) }\end{array}$ & $\begin{array}{l}\hat{x}^{(1)}(k) \\
=4322392.6250 e^{0.0008(k-1)} \\
-4318385.6250\end{array}$ & $\begin{array}{l}\hat{x}^{(0)}(k+1) \\
=3456.5281 e^{0.0008 k_{4}}\end{array}$ & 3492.5985 & 3495.3887 & 3498.1811 \\
\hline & production(million tons) & $\begin{array}{l}\hat{x}^{(1)}(k) \\
=62362.7203 e^{0.0261(k-1)} \\
-58415.7203\end{array}$ & $\begin{array}{l}\hat{x}^{(0)}(k+1) \\
=1559.4548 e^{0.0261 k}\end{array}$ & 2188.8204 & 2246.6534 & 2306.0144 \\
\hline \multirow{3}{*}{1} & yield(kg/hectare) & $\begin{array}{l}\hat{x}^{(1)}(k) \\
=1376183142.40 e^{0.0261(k-1)} \\
-137617.9196\end{array}$ & $\begin{array}{l}\hat{x}^{(0)}(k+1) \\
=3647.0659 e^{0.0261 k} .\end{array}$ & 5121.0678 & 5256.5439 & 5395.6039 \\
\hline & $\begin{array}{c}\text { Acreage } \\
\text { (one thousand hectares) }\end{array}$ & $\begin{array}{l}\hat{x}^{(1)}(k) \\
=-13359080.6667 e^{-0.0018(k-1)} \\
+13387935.6667\end{array}$ & $\begin{array}{l}\hat{x}^{(0)}(k+1) \\
=24067.5682 e^{-0.0018 k}\end{array}$ & 23503.0921 & 23460.2233 & 23417.4326 \\
\hline & production(million tons) & $\begin{array}{l}\hat{x}^{(1)}(k) \\
=-13359080.6667 e^{-0.0018(k-1)} \\
+13387935.6667\end{array}$ & $\begin{array}{l}\hat{x}^{(0)}(k+1) \\
=8670.4239 e^{0.0260 k} .\end{array}$ & 12163.5684 & 12484.4750 & 12813.8480 \\
\hline
\end{tabular}

Note: 0 -Shandong Province; 1 - the whole nation.

Table V The Errors between Actual Data and Forecast Data of Wheat Production in Shandong Province and the Whole Nation in 2012

\begin{tabular}{|c|c|c|c|}
\hline & Acreage(one thousand hectares) & $\begin{array}{c}\text { Production } \\
\text { (million tons) }\end{array}$ & $\begin{array}{c}\text { Yield } \\
(\mathrm{kg} / \mathrm{hectare})\end{array}$ \\
\hline
\end{tabular}




\begin{tabular}{|c|c|c|c|c|c|c|c|c|c|}
\hline data & actual data & Forecast data & relative error & $\begin{array}{c}\text { actual } \\
\text { data }\end{array}$ & Forecast data & relative error & actual data & Forecast data & $\begin{array}{c}\text { relative } \\
\text { error }\end{array}$ \\
\hline Shandong & 3625.87 & 3492.5985 & $3.68 \%$ & 2179.5 & 2188.8204 & $0.43 \%$ & 6011 & 6208.0036 & $3.28 \%$ \\
\hline Whole nation & 24412 & 23503.0921 & $3.73 \%$ & 12050 & 12163.5684 & $0.95 \%$ & 4937 & 5121.0678 & $3.73 \%$ \\
\hline
\end{tabular}

